\title{
M2H2: A Multimodal Multiparty Hindi Dataset For Humor Recognition in Conversations
}

\author{
Dushyant Singh Chauhan ${ }^{\dagger}$, Gopendra Vikram Singh ${ }^{\dagger}$, Navonil Majumder ${ }^{+}$, Amir Zadeh $^{\mp}$, \\ Asif Ekbal ${ }^{\dagger}$, Pushpak Bhattacharyya ${ }^{\dagger}$, Louis-philippe Morency $^{\mp}$, and Soujanya Poria ${ }^{+}$ \\ ${ }^{\dagger}$ Department of Computer Science \& Engineering \\ Indian Institute of Technology Patna, India \\ \{1821CS17, gopendra_1921CS15, asif, pb\}@iitp.ac.in \\ ${ }^{\mp}$ Language Technology Institute, Carnegie Mellon University, Pittsburgh, USA \\ \{abagherz, morency\}@cs. cmu.edu \\ ${ }^{+}$ISTD, Singapore University of Technology and Design, Singapore \\ \{navonil_majumder, sporia\} esutd.edu.sg
}

\begin{abstract}
Humor recognition in conversations is a challenging task that has recently gained popularity due to its importance in dialogue understanding, including in multimodal settings (i.e., text, acoustics, and visual). The few existing datasets for humor are mostly in English. However, due to the tremendous growth in multilingual content, there is a great demand to build models and systems that support multilingual information access. To this end, we propose a dataset for Multimodal Multiparty Hindi Humor (M2H2) recognition in conversations containing 6,191 utterances from 13 episodes of a very popular TV series "Shrimaan Shrimati Phir Se". Each utterance is annotated with humor/non-humor labels and encompasses acoustic, visual, and textual modalities. We propose several strong multimodal baselines and show the importance of contextual and multimodal information for humor recognition in conversations. The empirical results on $\mathrm{M} 2 \mathrm{H} 2$ dataset demonstrate that multimodal information complements unimodal information for humor recognition. The dataset and the baselines are available at http://www.iitp.ac.in/ ai-nlp-ml/resources.html and https://github.com/declare-lab/M2H2-dataset.
\end{abstract}

\section{CCS CONCEPTS}

- Computing methodologies; • Machine learning; • Machine learning approaches; $\bullet$ Neural networks;

\section{KEYWORDS}

Deep learning, Humor, Hindi dataset, Multimodal multiparty dataset

ACM Reference Format:

Dushyant Singh Chauhan $^{\dagger}$, Gopendra Vikram Singh ${ }^{\dagger}$, Navonil Majumder $^{+}$, Amir Zadeh $^{\mp}$, Asif Ekbal ${ }^{\dagger}$, Pushpak Bhattacharyya ${ }^{\dagger}$, Louis-philippe Morency $^{\mp}$, and Soujanya Poria ${ }^{+}$. 2021. M2H2: A Multimodal Multiparty Hindi Dataset For Humor Recognition in Conversations. In ICMI '21: 23rd

Permission to make digital or hard copies of all or part of this work for personal or classroom use is granted without fee provided that copies are not made or distributed for profit or commercial advantage and that copies bear this notice and the full citation on the first page. Copyrights for components of this work owned by others than ACM must be honored. Abstracting with credit is permitted. To copy otherwise, or republish, to post on servers or to redistribute to lists, requires prior specific permission and/or a fee. Request permissions from permissions@acm.org.

ICMI '21, October 18-22, 2021, Montreal, Canada

(C) 2021 Association for Computing Machinery.

ACM ISBN 978-1-4503-XXXX-X/18/06 . .\$15.00

https://doi.org/10.1145/1122445.1122456
ACM International Conference on Multimodal Interaction, Montreal, Canada. ACM, New York, NY, USA, 5 pages. https://doi.org/10.1145/1122445.1122456

\section{INTRODUCTION}

Humor [28] is defined as the nature of experiences to induce laughter and provide amusement. it can be implicit as well as explicit. Implicit humor may be expressed with neutral facial expressions and a polite tone, while explicit humor can be conveyed with laughter or funny facial expressions. Explicit humor may be easy to detect in comparison to implicit humor because of laughter or facial expression. So, while text $[1-3,5,9,10,14,22,23,29,34]$ alone may not always be enough to understand humor but if the utterance is multimodal in nature $[7,11,16,17,19-21,25-27,31]$ and is accompanied with a video of the facial expressions and tone of the speaker, it can be easily understood that the utterance contains humor.

Humor recognition is especially challenging in many Indian languages because of the following reasons: unavailability of resources and tools; morphological richness; free word order etc. In our current work, we focus on Hindi, one of the most popularly spoken languages in India. In terms of the number of speakers, Hindi ranks third ${ }^{1}$ all over in the world. In comparison to English, Hindi is much richer in terms of morphology. In Hindi, all the nouns and adjectives have genders, and the verb agrees in number and gender with them. While, English is free of that confounding factor of the gender of a common noun, and is also free of the further complication that the verb has to agree with the gender of the noun or pronoun governing it. These challenges motivated us to do some qualitative research work for Humor detection in Hindi.

In Hindi, there is no available dataset for humour detection (neither unimodal nor multimodal). In our work, we introduce a new dataset, named as $\mathrm{M} 2 \mathrm{H} 2$, which includes not only textual dialogues but also their corresponding visual and audio counterparts. The main contributions of our proposed research are as follows:

- We propose a dataset for Multimodal Multi-party Hindi Humor recognition in conversations. There are 6,191 utterances in the $\mathrm{M} 2 \mathrm{H} 2$ dataset;

\footnotetext{
https://currentaffairs.adda247.com/hindi-ranks-3rd-most-spoken-language-in-theworld/
} 
- We set up two strong baselines, viz., DialogueRNN [15] and bcLSTM [18] with MISA [12] for multimodal fusion. Both DialogueRNN and bcLSTM are contextual models.

- Empirical results on the $\mathrm{M} 2 \mathrm{H} 2$ dataset show the efficacy of multimodal information over only textual information for humor detection.

\section{DATASET}

We gathered samples from a famous TV show "Shrimaan Shrimati Phir Se"2 and annotated them manually. We make groups of these samples (utterances) based on their context into scenes. Each utterance in each scene consists of a label indicating humor of that utterance i.e., humor ${ }^{3}$ or non-humor. Besides, each utterance is also annotated with its speaker and listener information. In multiparty conversation, listener identification poses a great challenge. In our dataset, we define the listener as the party in the conversation to whom the speaker is replying. Each utterance in each scene is coupled with its context utterances, which are preceding turns by the speakers participating in the conversation. It also contains multiparty conversations that are more challenging to classify than dyadic variants. We show the dataset statistics in Table 1.

\begin{tabular}{|l|c|}
\hline Description & Statistic \\
\hline \#Scenes & 136 \\
\#Utterances & 6191 \\
Min \#Utterances per Scene & 8 \\
Max \#Utterances per Scene & 155 \\
\#Utterances per Scene & 45.52 \\
\#Words & 34472 \\
\#Unique words & 5454 \\
\#Humor class & 2089 \\
\#Non-humor class & 4102 \\
\#Speakers & 41 \\
Total duration & 4.46 Hours \\
\hline
\end{tabular}

Table 1: Dataset statistics.

\subsection{Challenges}

We crawled the video file and transcription file using YouTube for the TV show "Shrimaan Shrimati Phir Se". The downloaded Hindi transcription file had some errors. So, we hired transcribers, who were experts in the Hindi language. They wrote Hindi utterances based on the audio files.

As the context is based on the scenes, we divide the whole dataset into scenes based on its context. Each utterance in each scene consists of a humor label (i.e., humor or non-humor) and speaker and listener information.

\subsection{Annotation Guidelines}

We employ three Ph.D. students with high proficiency in Hindi and with prior experience in labeling Humor and Non-humor in conversational settings.

\footnotetext{
${ }^{2}$ Total of 4.46 hours in length

${ }^{3}$ There are $1 / 3^{r d}$ utterances which are humorous.
}

The guidelines for annotation, along with some examples (c.f. Figure 1), were explained to the annotators before starting the annotation process. The annotators were asked to annotate every utterance with humor/non-humor and corresponding speaker and listener information. We also annotated 20 annotations by ourselves and set it as a quality checker to evaluate the quality of the annotators. A majority voting scheme was used for selecting the final humor/nonhumor. We achieve an overall Fleiss' [8] kappa score of 0.84, which is considered to be reliable.

\section{BASELINE METHODS}

In this section, we provide strong benchmarks for $\mathrm{M} 2 \mathrm{H} 2$ dataset. We employ three strong baselines frameworks for humor detection.

\subsection{Strong Baseline for Multimodal Fusion: MISA}

MISA (Modality-Invariant and-Specific Representations for Multimodal Sentiment Analysis) [12] has two main stages: modality representation learning and modality fusion. It divides each modality into two sub-spaces. The first subspace is modality-invariant, in which representations from different modalities learn to share similarities and minimize the distance between them. The second subspace is modality-specific, which is unique to every modality and contains its distinguishing characteristics. These representations give a comprehensive perspective of multimodal data, which is utilized for fusion and task prediction. The model shows the state-of-the-art (SOTA) for MOSI [33] and MOSEI [32] datasets.

\subsection{Strong Conversation Classification Baseline \#1: DialogueRNN}

DialogueRNN [15] is a multi-party framework tailored for modelling emotions and sentiments in conversations. In DialogueRNN, they explained a novel recurrent neural network-based system that tracks of each party state during a discussion and uses this knowledge to classify emotions. It employs three levels of gated recurrent units (GRU) to represent the conversational context in order to accurately recognize emotions, intensity, and attitudes in a discussion. They showed the SOTA performance on two different datasets i.e., IEMOCAP [4] and AVEC [24].

\subsection{Strong Conversation Classification Baseline \#2: bcLSTM}

bcLstm [18] is a bidirectional contextual LSTM. Bi-directional LSTMs are formed by stacking two uni-directional LSTMs with opposing directions. As a result, an utterance can learn from utterances that come before and after it in the video which is of course context.

Leveraging the above three strong baselines, we formulate two setups, viz. MISA with DialogueRNN (MISA+DialogueRNN) and MISA with bcLSTM (MISA+bcLSTM). Here, MISA acts as a fusion model while DialogueRNN and bcLSTM are contextual models for conversation classification. DialogueRNN and bcLSTM have shown excellent performance in different conversation classification tasks such as Emotion Recognition Conversation. For MISA+DialogueRNN, we first pass multimodal inputs through MISA and obtain the fused features. These fused features are then 


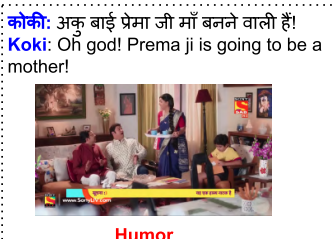

चिंट्र: लेकिन मेरे लिए तो एक ही पापा काफी है। Chintu: But for me, one father is enough.

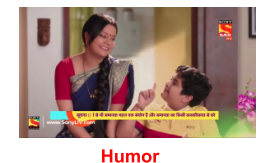

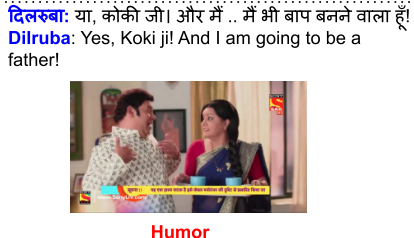

Humor

केश: गधे! ये अपने बच्चे का बाप बनेगा! Keshu: Donkey! He is going to be the father of his own child.

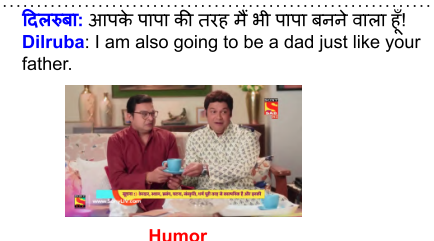

चिंट: ओ! मैं तो समझा मझे दो दो पापा मिलेंगे। Chintu: Oh! I thought I will be getting two fathers.

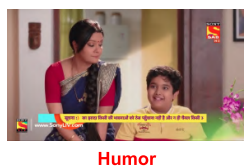

चिंट्: एक ऑफिस जाने के लिए और एक खाना बनाने के लिए Chintu: One to go to the office and one to cook.

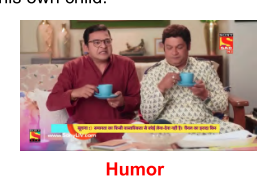

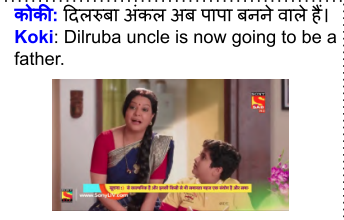

Non-humor

Figure 1: Some samples from dataset.

passed through DialogueRNN for humor classification. Similarly, for MISA+bcLSTM, We first pass multimodal inputs through MISA and obtain the fused features. Then, these fused features are passed through bcLSTM for humor classification.

\subsection{Feature Extraction}

For textual features, we take the pre-trained 300-dimensional Hindi fastText embeddings [13]. For visual feature extraction, we use 3DResNeXt- $101^{4}$ [30] which is pre-trained on Kinetics at a rate of 1.5 features per second and a resolution of 112 . While, we use openSMILE [6] for acoustic feature extraction. openSMILE ${ }^{5}$ can extract Low-Level Descriptors (LLD) and change them using different filters, functions, and transformations. We use a tonal low-level features group of openSMILE to extract the features.

\section{EXPERIMENTS AND ANALYSIS}

\subsection{Experimental Setup}

We evaluate our proposed model on the $\mathrm{M} 2 \mathrm{H} 2$ dataset. We perform five-fold cross-validation for experiments. Empirically, We take five ${ }^{6}$ utterances as context for a particular utterance.

We implement our proposed model on the Python-based PyTorch ${ }^{7}$ deep learning library. As the evaluation metric, we employ precision $(\mathrm{P})$, recall $(\mathrm{R})$, and $\mathrm{F} 1$-score $(\mathrm{F} 1)$ for humor recognition. We use Adam as an optimizer, Softmax as a classifier for humor detection, and the categorical cross-entropy as a loss function.

\subsection{Results and Analysis}

We evaluate our proposed architecture with all the possible input combinations (c.f. Table 2) i.e. unimodal $(T, A, V)$, bimodal $(T+V$, $T+A, A+V)$ and trimodal $(T+V+A)$.

For MISA+DialogueRNN, trimodal achieves the best precision of $71.21 \%\left(16.05^{8}\right.$ points $\uparrow$ and $9.51^{9}$ points $\left.\uparrow\right)$, recall of $72.11 \%$ (14.79

\footnotetext{
${ }^{4}$ https://github.com/kaiqiangh/extracting-video-features-ResNeXt

${ }^{5}$ https://github.com/audeering/opensmile

${ }^{6}$ Baseline models give the best result at five.

${ }^{7} \mathrm{https} / / /$ pytorch.org/

${ }^{8}$ maximum improvement over unimodal $(V)$

${ }^{9}$ maximum improvement over bimodal $(A+V)$
}

\begin{tabular}{|c|c|c|c||c|c|c|}
\hline \multirow{2}{*}{ Labels } & \multicolumn{3}{|c||}{ MISA+DialogueRNN } & \multicolumn{3}{c|}{ MISA+bcLSTM } \\
\cline { 2 - 7 } & $\mathbf{P}$ & $\mathbf{R}$ & $\mathbf{F 1}$ & $\mathbf{P}$ & $\mathbf{R}$ & F1 \\
\hline $\boldsymbol{T}$ & 67.11 & 68.21 & 67.65 & 66.27 & 66.51 & 66.38 \\
$\boldsymbol{A}$ & 57.91 & 59.10 & 58.52 & 57.51 & 58.92 & 58.21 \\
$\boldsymbol{V}$ & 55.16 & 57.32 & 56.74 & 53.13 & 55.21 & 54.19 \\
\hline $\boldsymbol{T +} \boldsymbol{V}$ & 70.03 & 70.61 & 70.31 & 67.74 & 68.89 & 68.31 \\
$\boldsymbol{T}+\boldsymbol{A}$ & 69.70 & 69.90 & 69.63 & 67.41 & 68.12 & 67.75 \\
$\boldsymbol{A + \boldsymbol { V }}$ & 61.70 & 63.21 & 62.44 & 59.61 & 61.23 & 60.40 \\
\hline $\boldsymbol{T +}+\boldsymbol{A}$ & $\mathbf{7 1 . 2 1}$ & $\mathbf{7 2 . 1 1}$ & $\mathbf{7 1 . 6 7}$ & $\mathbf{6 9 . 0 4}$ & $\mathbf{6 9 . 8 3}$ & $\mathbf{6 9 . 4 3}$ \\
\hline
\end{tabular}

Table 2: Experiment results for MISA+DialogueRNN and MISA+bcLSTM.

points $\uparrow$ and 8.9 points $\uparrow$ ) and $\mathrm{F} 1$-score of $71.67 \%$ (14.93 points $\uparrow$ and 9.23 points $\uparrow$ ). We observe that trimodal performs better than the unimodal and bimodal. We show the results in Table 2.

Similarly, for MISA+bcLSTM, trimodal achieves the best precision of $69.04 \%$ ( $15.91^{10}$ points $\uparrow$ and $9.43^{11}$ points $\left.\uparrow\right)$, recall of $69.83 \%$ (14.62 points $\uparrow$ and 8.6 points $\uparrow$ ) and F1-score of $69.43 \%$ (15.24 points $\uparrow$ and 9.03 points $\uparrow$ ). We observe that trimodal performs better than the unimodal and bimodal.

\subsection{Ablation Study}

We also perform an ablation study (c.f. Table 4) to show the efficacy of contextual models. We perform experiments on MISA (i.e., without contextual information). As per the result, we can see when MISA is used with contextual models (bcLSTM or DialogueRNN) it shows significant improvement rather than when it uses alone.

\subsection{Error Analysis}

In this section, we perform error analysis for our baseline system i.e, MISA+DialogueRNN. We take some samples which are correctly and incorrectly predicted by the baseline model to analyze the model's strengths and weaknesses.

\footnotetext{
$\overline{{ }^{10} \text { maximum improvement over unimodal }(V)}$

${ }^{11}$ maximum improvement over bimodal $(A+V)$
} 


\begin{tabular}{|c|c|c|c|c|c|}
\hline \multicolumn{6}{|c|}{ Correct Prediction } \\
\hline & \multirow[b]{2}{*}{ Hindi Utterances } & \multirow[b]{2}{*}{ English Utterances } & & \multicolumn{2}{|c|}{ Predicted } \\
\hline & & & Actual & $\begin{array}{l}\text { Unimodal } \\
\text { (T) }\end{array}$ & $\begin{array}{c}\text { Multimodal } \\
(\mathrm{T}+\mathrm{V}+\mathrm{A})\end{array}$ \\
\hline 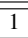 & अरे बुढापे में मैं तुम्हें खुद ले के जाता। & Oh in old age I would have taken you myself. & humor & "non-humor & humor \\
\hline 2 & $\begin{array}{l}\text { ये दिलरुबा कहता है ना कि पिछ़े जन्म में वो छोटूमल } \\
\text { मोटूमल करोडपति का एकलौता बेटा था, राइट? }\end{array}$ & $\begin{array}{l}\text { This Dilruba says that he was the only son of Chhotumal } \\
\text { Motumal Crorepati in his previous life, right? }\end{array}$ & non-humor & humor & non-humor \\
\hline 3 & स्टाफ वालों मेरा मतलब & Staff guys! I mean & humor & non-humor & humor \\
\hline 4 & कुछ, याद आया? कुछ, याद आया? & Remember anything? remember something? & non-humor & humor & non-humor \\
\hline 5 & जाते वक्त ऐसी अशुभ बारें नहीं करते। & Do not do such inauspicious things while leaving. & humor & non-humor & humor \\
\hline \multicolumn{6}{|c|}{ Incorrect Prediction } \\
\hline 1 & ये लोग मुझे बहुत मार रहे हैं। & These guys are beating me hard & humor & non-humor & non-humor \\
\hline 2 & हाय, टोटो! & hey, Toto! & non-humor & non-humor & humor \\
\hline 3 & ये पिंकी लाई। & Pinky brought this & humor & humor & non-humor \\
\hline 4 & अरे भाई, मेरी असली वाली गन यहीं छूट गैइ। & Hey brother, my original gun is left here & non-humor & humor & humor \\
\hline 5 & कौन सी असली है कौन सी नकली है। & which is real which is fake & non-humor & non-humor & humor \\
\hline
\end{tabular}

Table 3: Error analysis: Some correct and incorrect predicted samples.

\begin{tabular}{|l|c|c|c|}
\hline Setup & P & R & F1 \\
\hline \hline MISA+DialogueRNN & 71.21 & 72.11 & 71.67 \\
\hline MISA+bcLSTM & 69.04 & 69.83 & 69.43 \\
\hline MISA & 67.61 & 70.26 & 68.90 \\
\hline
\end{tabular}

Table 4: Ablation study

We show a few samples ${ }^{12}$ (c.f. Table 3 ) which are correctly predicted by the multimodal setup, but incorrectly predicted by the unimodal setup. For example, second utterance of the corrected prediction in Table 3, ये दिलरुबा कहता है ना कि पिछ्छले जन्म में वो छोट्रमल मोटूमल करोडपति का एकलौता बेटा था, राइट? (This Dilruba says that he was the only son of Chhotumal Motumal Crorepati in his previous life, right?) Gokhale is giving idea to Keshav with a polite tone and neutral facial expression. The actual label is non-humor but the unimodal model (text) predicts it as humorous instance while the multimodal model predicts the label correctly because it is clear from the acoustic and visual features that there were no humorous punches but only normal talk. We show some visual frames in Figure 2 which clearly show the neutral facial expression.

गोखले: ये दिलरुबा कहता है ना कि पिछले जन्म में वो छोटूमल मोट्मल करोड़पति का एकलौता बेटा था , राइट? Gokhale: This Dilruba says that he was the only son of Chhotumal Motumal Crorepati in his previous life, right?

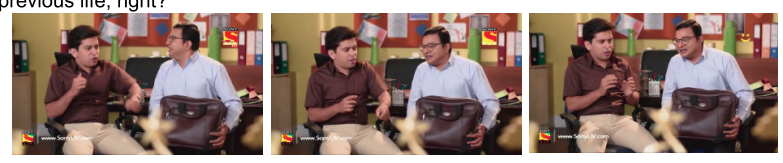

Figure 2: Some visual frames of second utterance of correct prediction in Table 3.

On the other hand, we also show some samples, where the multimodal model fails to predict the correct label. For example, first utterance of incorrect prediction in Table 3, ये लोग मुझे बहुत मार रहे हैं। (these guys are beating me hard) has the actual label as humor. But, the multimodal fails to predict the correct label because of the sad tone and the sad visual expression of Dilruba (दिलरुबा). We

\footnotetext{
${ }^{12}$ For the global audience, we also translate these Hindi utterances into English
}

show some visual frames in Figure 3 which clearly shows the sad facial expression of Dilruba.

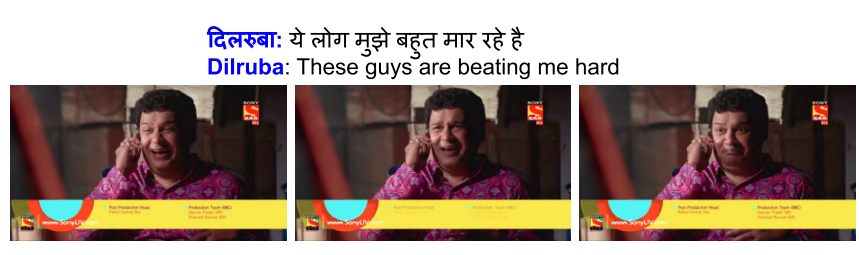

Figure 3: Some visual frames of first utterance of incorrect prediction in Table 3.

\section{CONCLUSION}

In this paper, we have presented a novel Multimodal Multi-party Hindi Humor (M2H2) recognition dataset for conversations (4.46 hours in length). We employed two strong baseline setups, viz. MISA with DialogueRNN and MISA with bcLSTM. MISA is a fusion model while DialogueRNN and bcLSTM are contextual models. We believe this dataset will also be useful as a useful resource for both conversational humor recognition and multimodal artificial intelligence. Empirical results on the $\mathrm{M} 2 \mathrm{H} 2$ dataset demonstrate that the multimodal baselines yield better performance over the unimodal framework.

In the future, we would like to extend our work towards the multi-party dialogue generation in Hindi with the help of humor and speaker information.

\section{ACKNOWLEDGEMENT}

Dushyant Singh Chauhan acknowledges the support of Prime Minister Research Fellowship (PMRF), Govt. of India. Asif Ekbal acknowledges the Young Faculty Research Fellowship (YFRF), supported by Visvesvaraya PhD scheme for Electronics and IT, Ministry of Electronics and Information Technology (Meit/8Y), Government of India, being implemented by Digital India Corporation (formerly Media Lab Asia). Soujanya Poria is supported by the SUTD SRG grant no. T1SRIS19149. 


\section{REFERENCES}

[1] Vladislav Blinov, Valeria Bolotova-Baranova, and Pavel Braslavski. 2019. Large dataset and language model fun-tuning for humor recognition. In Proceedings of the 57th annual meeting of the association for computational linguistics. 40274032 .

[2] San Bolkan, Darrin J Griffin, and Alan K Goodboy. 2018. Humor in the classroom: the effects of integrated humor on student learning. Communication Education 67, 2 (2018), 144-164.

[3] Maxwell Boykoff and Beth Osnes. 2019. A laughing matter? Confronting climate change through humor. Political Geography 68 (2019), 154-163.

[4] Carlos Busso, Murtaza Bulut, Chi-Chun Lee, Abe Kazemzadeh, Emily Mower, Samuel Kim, Jeannette N Chang, Sungbok Lee, and Shrikanth S Narayanan. 2008 IEMOCAP: Interactive emotional dyadic motion capture database. Language resources and evaluation 42, 4 (2008), 335.

[5] Santiago Castro, Luis Chiruzzo, Aiala Rosá, Diego Garat, and Guillermo Moncecchi. 2017. A crowd-annotated spanish corpus for humor analysis. arXiv preprint arXiv: 1710.00477 (2017).

[6] Florian Eyben, Martin Wöllmer, and Björn Schuller. 2010. Opensmile: the munich versatile and fast open-source audio feature extractor. In Proceedings of the 18th ACM international conference on Multimedia. ACM, 1459-1462.

[7] Fallianda Fallianda, Rani Yuni Astiti, and Zulvy Alivia Hanim. 2018. Analyzing Humor in Newspaper Comic Strips Using Verbal-Visual Analysis. Lingua Cultura 12, 4 (2018), 383-388.

[8] Joseph L. Fleiss. 1971. Measuring nominal scale agreement among many rater. Psychological Bulletin 76 (1971), 378-382.

[9] Noam Gal. 2019. Ironic humor on social media as participatory boundary work. New Media \& Society 21, 3 (2019), 729-749.

[10] Aparna Garimella, Carmen Banea, Nabil Hossain, and Rada Mihalcea. 2020. " Judge me by my size (noun), do you?'YodaLib: A Demographic-Aware Humor Generation Framework. arXiv preprint arXiv:2006.00578 (2020).

[11] Md Kamrul Hasan, Wasifur Rahman, Amir Zadeh, Jianyuan Zhong, Md Iftekhar Tanveer, Louis-Philippe Morency, et al. 2019. Ur-funny: A multimodal language dataset for understanding humor. arXiv preprint arXiv:1904.06618 (2019).

[12] Devamanyu Hazarika, Roger Zimmermann, and Soujanya Poria. 2020. MISA Modality-Invariant and-Specific Representations for Multimodal Sentiment Analysis. arXiv preprint arXiv:2005.03545 (2020).

[13] Armand Joulin, Edouard Grave, Piotr Bojanowski, Matthijs Douze, Hérve Jégou, and Tomas Mikolov. 2016. FastText.zip: Compressing text classification models. arXiv preprint arXiv:1612.03651 (2016).

[14] Ankush Khandelwal, Sahil Swami, Syed S Akhtar, and Manish Shrivastava. 2018. Humor detection in english-hindi code-mixed social media content: Corpus and baseline system. arXiv preprint arXiv:1806.05513 (2018).

[15] Navonil Majumder, Soujanya Poria, Devamanyu Hazarika, Rada Mihalcea, Alexander Gelbukh, and Erik Cambria. 2019. DialogueRNN: An Attentive RNN for Emotion Detection in Conversations. In The Thirty-Third AAAI Conference on Artificial Intelligence, AAAI 2019, The Thirty-First Innovative Applications of Artificial Intelligence Conference, IAAI 2019, The Ninth AAAI Symposium on Educational Advances in Artificial Intelligence, EAAI 2019, Honolulu, Hawaii, USA, January 27 - February 1, 2019, Vol. 33. 6818-6825.

[16] Nicole Mirnig, Gerald Stollnberger, Manuel Giuliani, and Manfred Tscheligi. 2017. Elements of humor: How humans perceive verbal and non-verbal aspects of humorous robot behavior. In Proceedings of the Companion of the 2017 ACM/IEEE International Conference on Human-Robot Interaction. 211-212.

[17] Anna Piata. 2020. Stylistic humor across modalities: The case of Classical Art Memes. Internet Pragmatics 3, 2 (2020), 174-201.

[18] Soujanya Poria, Erik Cambria, Devamanyu Hazarika, Navonil Majumder, Amir Zadeh, and Louis-Philippe Morency. 2017. Context-dependent sentiment analysis in user-generated videos. In Proceedings of the 55th Annual Meeting of the Association for Computational Linguistics (Volume 1: Long Papers), Vol. 1 873-883.

[19] Hannes Ritschel, Ilhan Aslan, David Sedlbauer, and Elisabeth André. 2019. Irony man: augmenting a social robot with the ability to use irony in multimodal communication with humans. (2019).

[20] Hannes Ritschel, Thomas Kiderle, Klaus Weber, Florian Lingenfelser, Tobias Baur, and Elisabeth André. 2020. Multimodal joke generation and paralinguistic personalization for a socially-aware robot. In International Conference on Practical Applications of Agents and Multi-Agent Systems. Springer, 278-290.

[21] Andy Jefferson Sabur, Retno Purwani Sari, and Tatan Tawami. 2020. Identification Of The Multimodal Structure Of Humor In An Animated Superhero Film. In International Conference on Language, Linguistics, and Literature (COLALITE) 2020.

[22] Sushmitha Reddy Sane, Suraj Tripathi, Koushik Reddy Sane, and Radhika Mamidi. 2019. Deep learning techniques for humor detection in Hindi-English code-mixed tweets. In Proceedings of the Tenth Workshop on Computational Approaches to Subjectivity, Sentiment and Social Media Analysis. 57-61.

[23] Tabea Scheel. 2017. Definitions, theories, and measurement of humor. In Humor at work in teams, leadership, negotiations, learning and health. Springer, 9-29.
[24] Björn Schuller, Michel Valster, Florian Eyben, Roddy Cowie, and Maja Pantic. 2012. Avec 2012: the continuous audio/visual emotion challenge. In Proceedings of the 14th ACM international conference on Multimodal interaction. 449-456.

[25] Kwangok Song, Kyle M Williams, Diane L Schallert, and Alina Adonyi Pruitt. 2021. Humor in multimodal language use: Students' Response to a dialogic, socialnetworking online assignment. Linguistics and Education 63 (2021), 100903.

[26] Camilla Vasquez and Erhan Aslan. 2021. "Cats be outside, how about meow": multimodal humor and creativity in an internet meme. Journal of Pragmatics 171 (2021), 101-117.

[27] Zhigailova Veronika. 2020. Multimodal Discourse Analysis of Humor in Picture Books for Children. (2020).

[28] Caleb Warren, Adam Barsky, and A Peter McGraw. 2018. Humor, comedy, and consumer behavior. Journal of Consumer Research 45, 3 (2018), 529-552.

[29] Orion Weller and Kevin Seppi. 2019. Humor detection: A transformer gets the last laugh. arXiv preprint arXiv:1909.00252 (2019).

[30] Saining Xie, Ross Girshick, Piotr Dollár, Zhuowen Tu, and Kaiming He. 2017. Aggregated residual transformations for deep neural networks. In Proceedings of the IEEE conference on computer vision and pattern recognition. 1492-1500.

[31] Zixiaofan Yang, Lin Ai, and Julia Hirschberg. 2019. Multimodal Indicators of Humor in Videos. In 2019 IEEE Conference on Multimedia Information Processing and Retrieval (MIPR). IEEE, 538-543.

[32] Amir Zadeh, Paul Pu Liang, Soujanya Poria, Erik Cambria, and Louis-Philippe Morency. 2018. Multimodal Language Analysis in the Wild: CMU-MOSEI Dataset and Interpretable Dynamic Fusion Graph. In Proceedings of the 56th Annual Meeting of the Association for Computational Linguistics (Volume 1: Long Papers) (Melbourne, Australia). Association for Computational Linguistics, 2236-2246. http://aclweb.org/anthology/P18-1208

[33] A. Zadeh, R. Zellers, E. Pincus, and L. P. Morency. 2016. Multimodal Sentiment Intensity Analysis in Videos: Facial Gestures and Verbal Messages. IEEE Intelligent Systems 31, 6 (Nov 2016), 82-88. https://doi.org/10.1109/MIS.2016.94

[34] Zhenjie Zhao, Andrew Cattle, Evangelos Papalexakis, and Xiaojuan Ma. 2019. Embedding lexical features via tensor decomposition for small sample humor recognition. In Proceedings of the 2019 Conference on Empirical Methods in Natural Language Processing and the 9th International Joint Conference on Natural Language Processing (EMNLP-IJCNLP). 